\title{
Numerical solution of the 2D Poisson equation on an irregular domain with Robin boundary conditions
}

\author{
Z. Jomaa ${ }^{1} \quad$ C. Macaskill ${ }^{2}$
}

(Received 15 August 2008; revised 6 November 2008)

\begin{abstract}
We describe a 2D finite difference algorithm for inverting the Poisson equation on an irregularly shaped domain, with mixed boundary conditions, with the domain embedded in a rectangular Cartesian grid. We give both linear and quadratic boundary treatments and derive 1D error expressions for both cases. The linear approach uses a five point formulation and is first order accurate while the quadratic treatment uses a nine point stencil and is second order accurate. The key aspect of the quadratic treatment is the use of a suitably chosen directional derivative to find the second order accurate approximation to the normal derivative at the boundary.
\end{abstract}

http://anziamj.austms.org.au/ojs/index.php/ANZIAMJ/article/view/1453 gives this article, (c) Austral. Mathematical Soc. 2008. Published November 21, 2008. ISSN 1446-8735. (Print two pages per sheet of paper.) 


\section{Contents}

1 Introduction

C414

2 Mathematical formulation

$2.12 \mathrm{D}$ quadratic boundary treatment . . . . . . . . . . .

2.2 1D error analysis of the Robin boundary condition problem

3 Numerical results

4 Conclusion

C425

References

\section{Introduction}

We discuss an embedding method for numerical solution of the 2D Poisson equation on an irregular domain subject to Robin boundary conditions. The crucial feature of the formulation is the discretisation of the normal derivative at the irregular boundary. Our approach for treatment of the normal derivative is similar to that of Greenspan [5] who uses Taylor expansions to obtain a second order accurate discretisation. By contrast, Liu, Fedkiw and Kang [8] develop a first order accurate symmetric discretisation of the variable coefficient Poisson equation in the presence of an irregular interface. Bramble and Hubbard [2] present first order and second order discretisations of the normal derivative, but use of the tangential derivative of the boundary condition is essential in their formulation. However, the resultant coefficient matrix is an M-matrix [3] which ensures convergence when iterative schemes are used to carry out the inversion. More recently, Bouchon and Peichl [1] developed a second order discretisation of the normal derivative, avoiding the use of the tangential derivative but still obtaining an M-matrix formulation. Our present approach also avoids the use of tangential derivative, but does 
not lead to an M-matrix. However, the present scheme is more compact and also provides a clear extension to the 3D case.

In the present scheme, the Poisson equation is discretised at each grid point. For grid points away from the boundary the algorithm uses the standard five point discretisation for the second derivative. For internal grid points next to the boundary, we use either the Shortley-Weller (quadratic boundary fit) approach [9] or the linear boundary fit of Collatz [4]. At the boundaries, correspondingly, the normal derivative is discretised to first order for the linear case and second order for the quadratic case respectively. To gain insight into the nature of the overall discretisation error introduced, we develop explicit error expressions for both boundary treatments for the 1D case. By contrast with the Dirichlet problem, the higher dimensional Poisson solver cannot be built by repeated application in each dimension of the 1D solver, so that the predictive capability of the quantitative error in higher dimensions using the 1D error expressions is limited. However, we find that the qualitative behaviour obtained using these expressions is confirmed in $2 \mathrm{D}$ numerical tests.

\section{Mathematical formulation}

Our aim is to solve the Poisson equation for $\psi$,

$$
\nabla^{2} \psi=f(x, y)
$$

on an irregular domain $\Omega$ with Robin boundary conditions,

$$
\beta \psi_{\mathrm{n}}+\psi=\gamma
$$

on the boundary $\partial \Omega$ and where $\beta=\beta(x, y)$ and $\gamma=\gamma(x, y)$ are given. 


\section{$2.12 \mathrm{D}$ quadratic boundary treatment}

Consider the North-East corner point $(i, j)$ without loss of generality (see Figure 1$)$. We discretise the Poisson equation at $(i, j)$ subject to boundary conditions of the form (2) at boundary points 1 and 2. Without loss of generality, let $\Delta x=\Delta y=\Delta$. The second order accurate Shortley-Weller discretisation at $(\boldsymbol{i}, \boldsymbol{j})$ is

$$
\begin{aligned}
& \frac{2}{\Delta^{2}}\left[-\frac{1}{\left(1-\alpha_{E}\right)\left(2-\alpha_{E}\right)} \psi_{E}+\left(\frac{1}{1-\alpha_{E}}+\frac{1}{1-\alpha_{N}}\right) \psi_{i, j}-\frac{1}{2-\alpha_{E}} \psi_{i-1, j}\right. \\
& \left.-\frac{1}{2-\alpha_{N}} \psi_{i, j-1}-\frac{1}{\left(1-\alpha_{N}\right)\left(2-\alpha_{N}\right)} \psi_{N}\right]=f_{i, j},
\end{aligned}
$$

where $\alpha_{E}$ and $\alpha_{N}$ determine the distance of the two boundary points from the grid (see Figure 1).

To complete the formulation, expressions for boundary values $\psi_{\mathrm{E}}$ and $\psi_{\mathrm{N}}$ are required. (Contrast this with the Dirichlet case, where these values are given.) In order to do this the normal derivative at the boundary must be approximated. Consider for instance the normal derivative at the Eastern boundary point (boundary point 1)

$$
\psi_{n}^{E}=\psi_{x}^{E} n_{x}^{E}+\psi_{y}^{E} n_{y}^{E},
$$

with $\mathbf{n}^{\mathrm{E}}=\left(\mathbf{n}_{x}^{\mathrm{E}}, \mathbf{n}_{y}^{\mathrm{E}}\right)$ the outward normal at that point. Here the $\boldsymbol{x}$-component of $\psi_{n}^{\mathrm{E}}$ aligns with the grid and therefore is approximated directly in terms of $\psi_{E}, \psi_{i, j}$ and $\psi_{i-1, j}$ as

$$
\psi_{x}^{E}=\frac{1}{\Delta}\left[-\frac{2-\alpha_{E}}{1-\alpha_{E}} \psi_{i, j}+\frac{1-\alpha_{E}}{2-\alpha_{E}} \psi_{i-1, j}+\frac{3-2 \alpha_{E}}{\left(1-\alpha_{E}\right)\left(2-\alpha_{E}\right)} \psi_{E}\right] .
$$

However, a different approach is needed for the $y$-component.

The derivative $\psi_{E}^{\prime}$ in the direction of the line passing through the grid point $(i-1, j-1)$ and boundary point 1 is resolved into two components in 


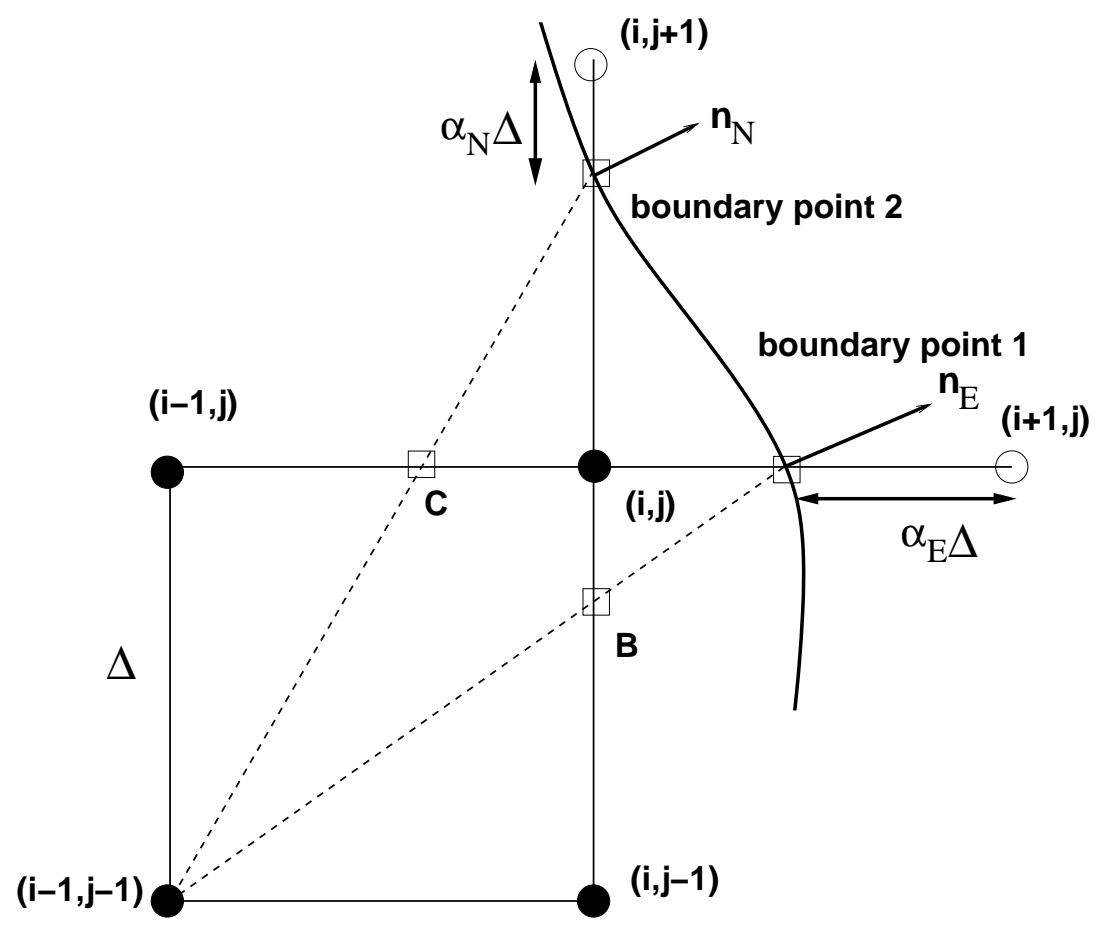

FiguRE 1: Diagram of a North-East corner point, filled circle are interior points, open circle are exterior points, the boxes on the solid curve are boundary points, while the point $\mathrm{B}$ and $\mathrm{C}$ are used temporarily to determine the second order accurate formulation. 
the $x$ - and $y$-directions. Substituting the $y$-component of the derivative at $E$ in the normal derivative leads to an expression in terms of $\psi_{n}^{\mathrm{E}}, \psi_{x}^{\mathrm{E}}$ and $\psi_{\mathrm{E}}^{\prime}$, that is,

$$
\sqrt{1+\left(2-\alpha_{\mathrm{E}}\right)^{2}} \psi_{\mathrm{E}}^{\prime}=\left(2-\alpha_{\mathrm{E}}-\frac{n_{x}^{\mathrm{E}}}{n_{y}^{\mathrm{E}}}\right) \psi_{x}^{\mathrm{E}}+\frac{1}{n_{\mathrm{y}}^{\mathrm{E}}} \psi_{n}^{\mathrm{E}} .
$$

But $\psi_{\mathrm{E}}^{\prime}$ is expressed in terms of $\psi_{i-1, j-1}$ and $\psi_{\mathrm{E}}$ and the value at the temporary point $\psi_{\mathrm{B}}$ as

$$
\sqrt{1+\left(2-\alpha_{E}\right)^{2}} \Delta \psi_{E}^{\prime}=\left(1-\alpha_{E}\right) \psi_{i-1, j-1}-\frac{\left(2-\alpha_{E}\right)^{2}}{1-\alpha_{E}} \psi_{B}+\frac{3-2 \alpha_{E}}{1-\alpha_{E}} \psi_{E} .
$$

Then we eliminate $\psi_{\mathrm{B}}$ by expanding in terms of $\psi_{\mathrm{N}}$ and the two internal values $\psi_{i, j}$ and $\psi_{i, j-1}$ :

$$
\begin{aligned}
\frac{\left(2-\alpha_{E}\right)^{2}}{1-\alpha_{E}} \psi_{B} & =-\frac{1}{\left(1-\alpha_{N}\right)\left(2-\alpha_{N}\right)} \psi_{N}+\left(\frac{1}{1-\alpha_{E}}+\frac{2-\alpha_{N}}{1-\alpha_{N}}\right) \psi_{i, j} \\
& +\left(1-\alpha_{E}+\frac{1-\alpha_{N}}{2-\alpha_{N}}\right) \psi_{i, j-1} .
\end{aligned}
$$

Combining all these results in the boundary condition at boundary point 1 leads to an equation for $\psi_{E}$ and $\psi_{N}$ in terms of the four internal values $\psi_{i, j}$, $\psi_{i-1, j}, \psi_{i, j-1}$ and $\psi_{i-1, j-1}$ :

$$
\begin{aligned}
& {\left[1+\frac{\beta_{E}\left(3-2 \alpha_{E}\right)}{\Delta\left(1-\alpha_{E}\right)\left(2-\alpha_{E}\right)} n_{x}^{E}\right] \psi_{E}+\frac{\beta_{E} n_{y}^{E}}{\Delta\left(1-\alpha_{N}\right)\left(2-\alpha_{N}\right)} \psi_{N}} \\
& =\frac{\beta_{E}}{\Delta}\left\{\left[\frac{2-\alpha_{E}}{1-\alpha_{E}} n_{x}^{E}-\left(2-\alpha_{E}-\frac{1}{1-\alpha_{N}}\right) n_{y}^{E}\right] \psi_{i, j}-\left(1-\alpha_{E}\right) n_{y}^{E} \psi_{i-1, j-1}\right. \\
& \left.-\left[\frac{1-\alpha_{E}}{2-\alpha_{E}} n_{x}^{E}-\left(1-\alpha_{E}\right) n_{y}^{E}\right] \psi_{i-1, j}+\left[1-\alpha_{E}+\frac{1-\alpha_{N}}{2-\alpha_{N}}\right] n_{y}^{E} \psi_{i, j-1}\right\}+\gamma_{E} .
\end{aligned}
$$

Similarly, consideration of the boundary condition at the Northern boundary point (boundary point 2 ) gives a second relation between $\psi_{\mathrm{E}}$ and $\psi_{\mathrm{N}}$. 
The resulting equations are solved simultaneously to give expressions for $\psi_{\mathrm{E}}$ and $\psi_{\mathrm{N}}$ in terms of the four internal grid points marked with solid circles in Figure 1. Substituting this result in the Shortley-Weller formula (3) completes the discretisation of the Poisson equation at the point $(i, j)$ subject to the two Robin boundary conditions at boundary points 1 and 2. The 2D Taylor series approach of Greenspan [5] for the approximation for the normal derivative leads to the same result as equation (9).

Finally, the 3D extension of our approach follows directly from the 2D scheme above. For example, considering the normal derivative at a boundary point where only the $x$-component aligns with the grid, we use the $2 \mathrm{D}$ approach in the $x y$-plane by considering a directional derivative to eliminate the $y$-components of the normal derivative and similarly use a directional derivative in the $x z$-plane to eliminate the $z$-component of the normal derivative. For the general case of three such boundary points adjacent to an internal grid point, this will give rise to a $3 \times 3$ matrix system to be inverted for the values of $\psi$ at these three boundary points. With more effort, a 3D Taylor series expansion gives the same result [6].

\subsection{D error analysis of the Robin boundary condition problem}

This analysis follows the work of Jomaa and Macaskill [7]. Consider the 1D Poisson equation on an interval $\left(x_{L}, x_{R}\right)$ with Robin boundary conditions applied. The error $\xi=\psi-\psi^{e}$, where $\psi^{e}$ is the exact solution, satisfies

$$
\mathrm{L} \xi=\tau, \quad-\left.\beta_{\mathrm{L}} \frac{\mathrm{d} \xi}{\mathrm{d} x}\right|_{x=x_{\mathrm{L}}}+\xi_{\mathrm{L}}=0,\left.\quad \beta_{\mathrm{R}} \frac{\mathrm{d} \xi}{\mathrm{d} x}\right|_{x=x_{\mathrm{R}}}+\xi_{\mathrm{R}}=0,
$$

where $\alpha_{L}$ and $\alpha_{R}$ play analogous roles to the $2 \mathrm{D}$ case, $\tau$ is the truncation error, $\mathrm{L}$ is the discrete second derivative, and $\beta_{\mathrm{L}}$ and $\beta_{\mathrm{R}}$ are constants. Solving (10) 
for $\xi$ using the quadratic boundary treatment, we obtain

$$
\begin{aligned}
& \xi_{k}=\Delta^{2}\left[\left(k-\alpha_{L}+\frac{\beta_{L}}{\Delta}\right) \frac{H_{M-1 / 2}}{\Delta}-\sum_{j=2}^{M-1}\left(j-\alpha_{L}+\frac{\beta_{L}}{\Delta}\right) \tau_{j}-\sum_{j=k+1}^{M-1}(k-j) \tau_{j}\right. \\
& \left.-\frac{\left(1-\alpha_{L}\right)\left(2-\alpha_{L}\right) \Delta+\beta_{L}\left(3-2 \alpha_{L}\right)}{2 \Delta} \tau_{1}\right] \text { for } 1 \leq k \leq M-1,
\end{aligned}
$$

with

$$
\begin{aligned}
& \mathrm{H}_{M-1 / 2}=\Delta^{2}\left\{\sum_{j=2}^{M-1}\left(j-\alpha_{L}+\frac{\beta_{L}}{\Delta}\right) \tau_{j}+\frac{\alpha_{R}\left(1-\alpha_{R}\right) \Delta-\beta_{R}\left(1-2 \alpha_{R}\right)}{2 \Delta} \tau_{M-1}\right. \\
& \left.+\frac{\left(1-\alpha_{L}\right)\left(2-\alpha_{L}\right)+\beta_{L}\left(3-2 \alpha_{L}\right)}{2 \Delta} \tau_{1}\right\} \times\left[\left(M-\alpha_{L}-\alpha_{R}\right) \Delta+\beta_{L}+\beta_{R}\right]^{-1}
\end{aligned}
$$

and

$$
\begin{gathered}
\tau_{1}=-\frac{\Delta}{3} \alpha_{\mathrm{L}} \psi_{1}^{\prime \prime \prime}-\frac{\beta_{\mathrm{L}}\left(1-\alpha_{\mathrm{L}}\right)\left(2-\alpha_{\mathrm{L}}\right) \Delta}{3\left[\left(1-\alpha_{\mathrm{L}}\right)\left(2-\alpha_{\mathrm{L}}\right) \Delta+\beta_{\mathrm{L}}\left(3-2 \alpha_{\mathrm{L}}\right)\right]} \psi_{\mathrm{L}}^{\prime \prime \prime}, \\
\tau_{M-1}=\frac{\Delta}{3} \alpha_{\mathrm{R}} \psi_{M-1}^{\prime \prime \prime}+\frac{\beta_{\mathrm{R}}\left(1-\alpha_{\mathrm{R}}\right)\left(2-\alpha_{R}\right) \Delta}{3\left[\left(1-\alpha_{\mathrm{R}}\right)\left(2-\alpha_{\mathrm{R}}\right) \Delta+\beta_{\mathrm{R}}\left(3-2 \alpha_{\mathrm{R}}\right)\right]} \psi_{\mathrm{R}}^{\prime \prime \prime}, \\
\tau_{k}=-\frac{(\Delta)^{2}}{12} \psi_{\mathrm{k}}^{(4)}, \quad 2 \leq \mathrm{k} \leq \mathrm{M}-2 .
\end{gathered}
$$

Turning to the linear boundary treatment, the solution of equation (10) for $\xi$ is

$$
\begin{aligned}
\xi_{k} & =\Delta\left[\left(\frac{\beta_{L}+\left(k-\alpha_{L}\right) \Delta}{\left(M-\alpha_{L}-\alpha_{R}\right) \Delta+\beta_{L}+\beta_{R}}-1\right) \sum_{j=1}^{M-1}\left(\beta_{L}+\left(j-\alpha_{L}\right) \Delta\right) \tau_{j}\right. \\
& \left.-\Delta \sum_{j=k+1}^{M-1}(k-j) \tau_{j}\right] \quad \text { for } \quad 1 \leq k \leq M-1,
\end{aligned}
$$


where

$$
\begin{gathered}
\tau_{1}=\frac{\alpha_{\mathrm{L}}}{2} \psi_{1}^{\prime \prime}-\frac{\beta_{\mathrm{L}}\left(1-\alpha_{\mathrm{L}}\right)}{2\left(\beta_{\mathrm{L}}+\left(1-\alpha_{\mathrm{L}}\right) \Delta\right)} \psi_{\mathrm{L}}^{\prime \prime}, \\
\tau_{M-1}=\frac{\alpha_{\mathrm{R}}}{2} \psi_{M-1}^{\prime \prime}-\frac{\beta_{\mathrm{R}}\left(1-\alpha_{\mathrm{R}}\right)}{2\left(\beta_{\mathrm{R}}+\left(1-\alpha_{\mathrm{R}}\right) \Delta\right)} \psi_{\mathrm{R}}^{\prime \prime} .
\end{gathered}
$$

For both linear and quadratic boundary treatments, the internal truncation errors $\tau_{i}, i=2, \ldots, M-2$, are given by equation (15) with $\mathcal{O}\left(\Delta^{2}\right)$ accuracy. However, the boundary truncation errors $\tau_{1}$ and $\tau_{M-1}$ are $\mathcal{O}(1)$ for the linear boundary treatment and $\mathcal{O}(\Delta)$ for the quadratic boundary treatment respectively. In the Dirichlet case $(\beta=0)$ the corresponding boundary errors $\xi_{1}$ and $\xi_{M-1}$ are then $\mathcal{O}\left(\Delta^{2}\right)$ for the linear boundary treatment and $\mathcal{O}\left(\Delta^{3}\right)$ for the quadratic boundary treatment, as is to be expected since inverting the Poisson operator corresponds to integrating twice. This means that when taken together with the internal errors of $\mathcal{O}\left(\Delta^{2}\right)$ either approach gives rise to a uniformly $\mathcal{O}\left(\Delta^{2}\right)$ method, although the coefficient of error is significantly larger with the linear boundary treatment [7].

By contrast, with Robin boundary conditions, the boundary and internal errors $\xi_{k}$ are $\mathcal{O}(\beta \Delta)$ for the linear boundary treatment and $\mathcal{O}\left(\beta \Delta^{2}\right)$ for the quadratic boundary treatment. Therefore, with the restriction that $\beta \ll$ $M=\mathcal{O}(1 / \Delta)$, the linear case gives rise to a uniformly $\mathcal{O}(\Delta)$ method, while the quadratic approach gives a uniformly $\mathcal{O}\left(\Delta^{2}\right)$ method. Thus in practice the quadratic treatment is preferred for Robin boundary conditions.

\section{$3 \quad$ Numerical results}

This section presents numerical results for two test cases. In Figure 2, we treat a representative 1D problem and demonstrate that the analytical error estimates are quantitatively accurate in this case (a range of other test cases 

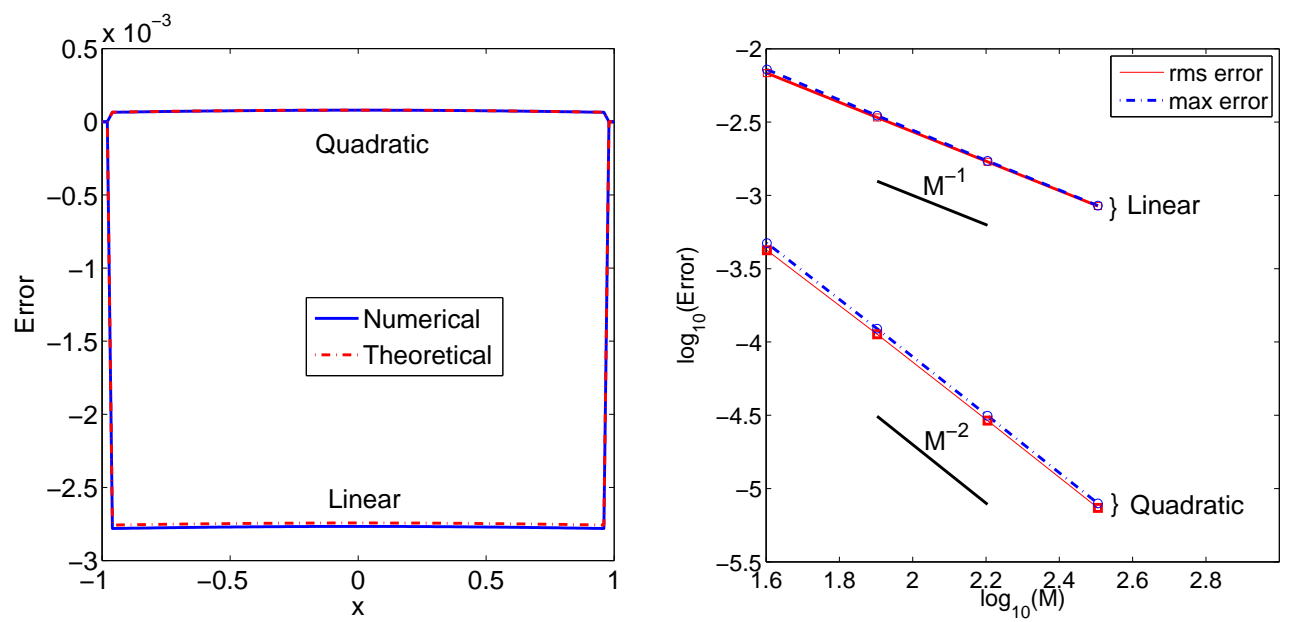

Figure 2: The error in solving the $1 \mathrm{D}$ problem $\mathrm{d}^{2} \psi / \mathrm{d} x^{2}=-\cos x$, with boundary conditions $\mathrm{d} \psi / \mathrm{d} x+\psi=\gamma$ for $x= \pm(1-5 \Delta / 4)$ respectively, with $\gamma=\cos x \mp \sin x$. In the left-hand panel the numerical errors for $M=$ 100 are shown with solid curves for both the quadratic and linear boundary treatments and compared with the corresponding analytic error expressions (dash-dotted lines) for the quadratic (11), or linear (16) cases respectively. The RMS errors (solid lines) and maximum absolute errors (dash-dotted lines) for the linear and quadratic boundary treatments are displayed in the righthand panel.

show similar agreement). We then consider in Figures 3, 4 and 5 a 2D problem that shows qualitatively similar behaviour. For all results shown here we used direct inversion of the coefficient matrix. However iterative methods can also be used without any problems, although the coefficient matrices are not M-matrices. We used the conjugate gradient squared iteration method for sparse matrices as implemented in MATLAB, cgs.m, for a range of $2 \mathrm{D}$ problems and no difficulties were encountered.

The left-hand panel of Figure 2 shows the numerical error (solid lines) 


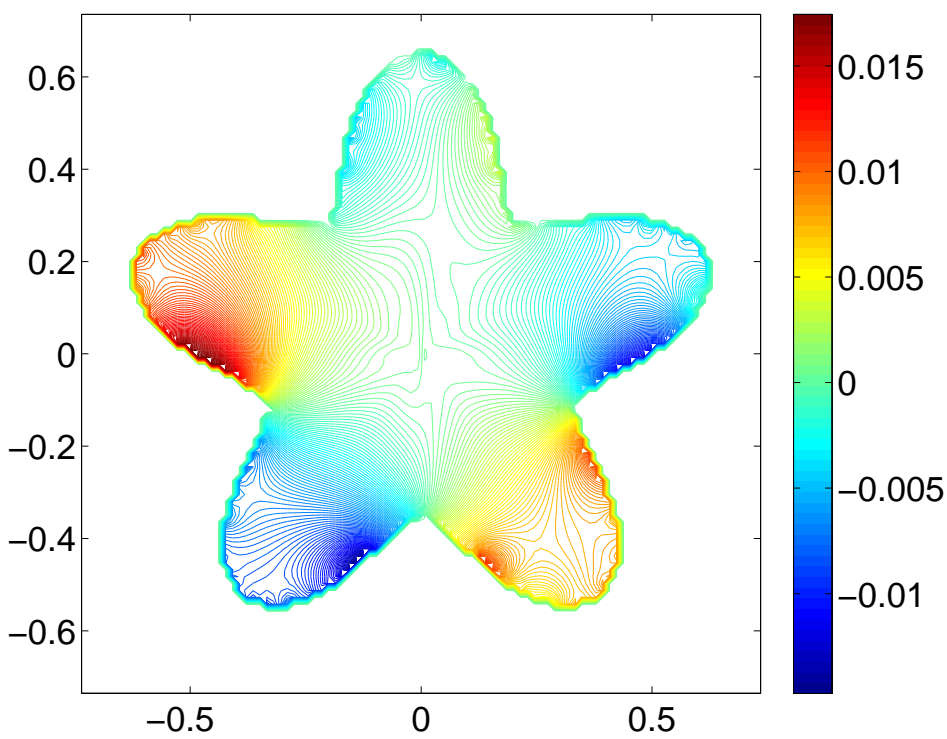

Figure 3: Contour of the error for the linear boundary treatment for the $2 \mathrm{D}$ problem $\nabla^{2} \psi=-5 \cos 3 \theta-40 \mathrm{r} \cos 7 \theta,(M+1) \Delta=1.5$ and $M=100$ subject to $\partial \psi / \partial n+\psi=\gamma$ on the boundary of the five-leaf shape described by $r=0.5+0.15 \cos 5 \theta$ and with $\psi=0$ elsewhere. $\gamma$ is given by the exact boundary values of the left hand side of the boundary conditions.

compared with the theoretical error (dash-dotted lines) for both quadratic and linear boundary treatments of the case with the exact solution $\psi=\cos x$, for $M=100$. For the same test problem the right panel shows the RMS error (solid lines) and maximum absolute error (dash-dotted lines) for both linear and quadratic boundary treatments with $M=40,80,160$ and 320. Errors converge like $\mathcal{O}\left(\Delta^{2}\right)$ for the quadratic boundary treatment as against $\mathcal{O}(\Delta)$ for the linear boundary treatment, as expected from the error analysis.

Figures 3 and 4 show contours of the error for the linear and quadratic boundary treatments respectively, for the Poisson equation $\nabla^{2} \psi=-5 \cos 3 \theta-$ $40 \mathrm{r} \cos 7 \theta$ on a 'five-leaf' shape, with Robin boundary conditions where $\beta=1$ 


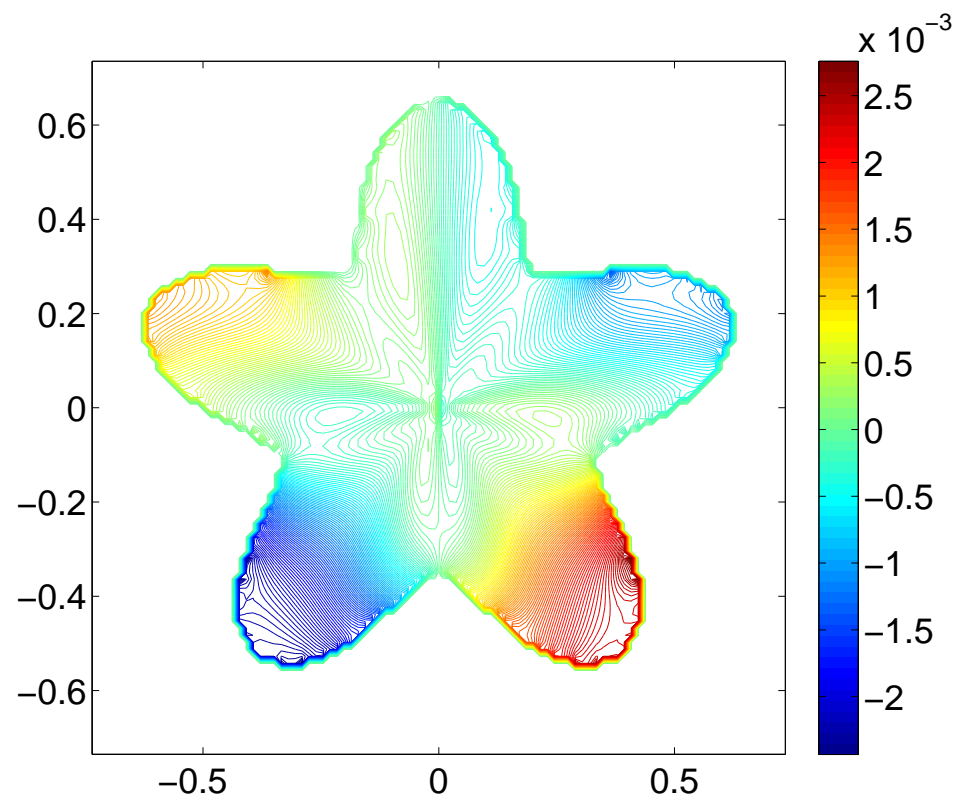

Figure 4: Contour of the error for the quadratic boundary treatment for the $2 \mathrm{D}$ problem $\nabla^{2} \psi=-5 \cos 3 \theta-40 \mathrm{r} \cos 7 \theta,(M+1) \Delta=1.5$ and $M=100$ subject to $\partial \psi / \partial \mathrm{n}+\psi=\gamma$ on the boundary of the five-leaf shape described by $r=0.5+0.15 \cos 5 \theta$ and with $\psi=0$ elsewhere. $\gamma$ is given by the exact boundary values of the left hand side of the boundary conditions. 
and using $M=100$. Outside the irregular shape we set $\psi=0$. The domain is embedded in a square domain of side length 1.5. The maximum absolute error for the linear case is approximately six times larger than the maximum absolute error for the quadratic case: there is no particular tendency for errors to be maximal at the boundary. The left-hand panel of Figure 5 compares the RMS errors (solid lines) and maximum absolute errors (dash-dotted lines) for the same problem for a range of values of $M$ for both the linear and quadratic boundary treatments. The right-hand panel shows corresponding results for $\beta=10$ (note how the error scales with $\beta$, as predicted by the $1 \mathrm{D}$ analysis). In summary, the observed errors for this $2 \mathrm{D}$ problem show similar convergence properties to the 1D cases, and we found similar results for a broad range of test problems. By contrast with similar problems with Dirichlet boundary conditions, one must necessarily use a quadratic boundary treatment in order to obtain $\mathcal{O}\left(\Delta^{2}\right)$ convergence. The 1D analysis shows that with Robin boundary conditions, a linear boundary treatment gives uniform $\mathcal{O}(\Delta)$ error as opposed to the $\mathcal{O}\left(\Delta^{2}\right)$ error found with a quadratic treatment.

\section{Conclusion}

We presented a simple geometric derivation of a second order accurate solution for the Poisson equation on an irregular domain subject to Robin boundary conditions. A 1D error analysis suggests that the quadratic boundary treatment method leads to a uniformly $\mathcal{O}\left(\Delta^{2}\right)$ accurate method as opposed to $\mathcal{O}(\Delta)$ for the linear boundary treatment method. This agrees with numerical results for $2 \mathrm{D}$ test cases. Thus when an embedding method is used to deal with irregular boundaries, it is necessary to use the quadratic approach in order to match the standard $\mathcal{O}\left(\Delta^{2}\right)$ internal error. This is in contrast to the Dirichlet problem, where a linear boundary treatment is sufficient to maintain the $\mathcal{O}\left(\Delta^{2}\right)$ error. Finally, the extension of the present scheme to $3 \mathrm{D}$ is straightforward. 

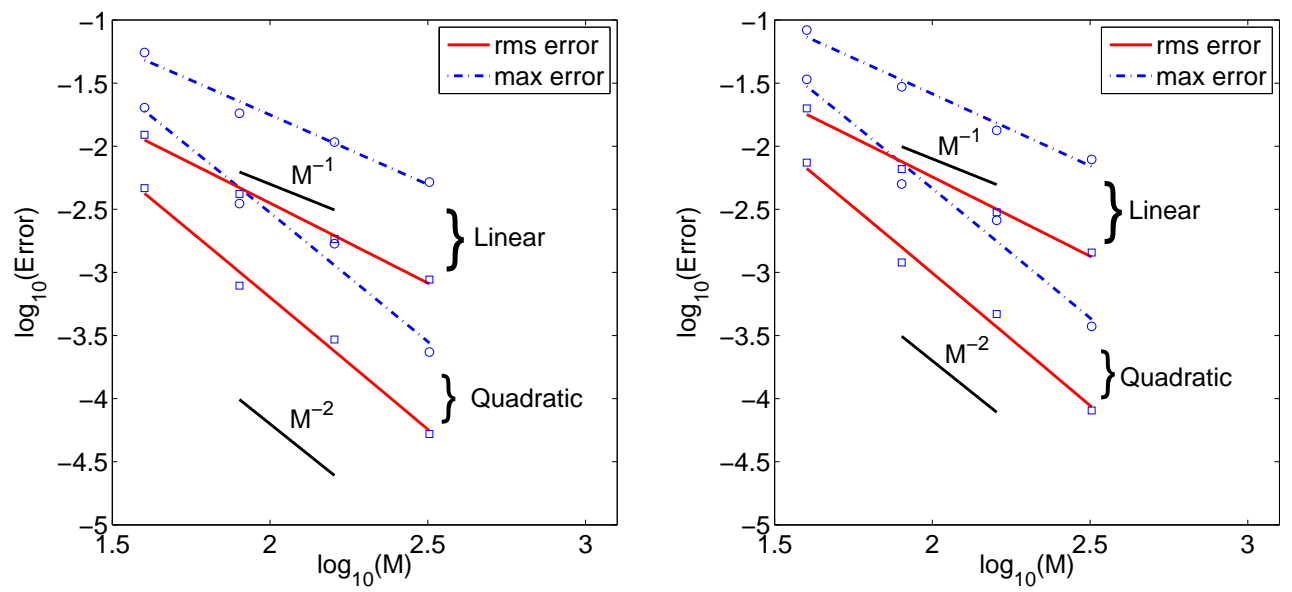

FigURE 5: RMS and maximum absolute errors for the case treated in Figures 3 and 4 for both linear and quadratic boundary treatments. The lefthand panel shows the RMS errors (solid lines) and maximum absolute errors (dash-dotted lines) for the case $\beta=1$, while the right-hand panel shows the case $\beta=10$. 


\section{References}

[1] F. Bouchon and G. H. Peichl, A second-order immersed interface technique for an elliptic Neumann problem, Numer. Methods Partial Differential Equations. 23 (2007), no. 2, 400-420. doi:10.1002/num.20187 C414

[2] J. H. Bramble and B. E. Hubbard, Approximation of solutions of mixed boundary value problems for Poisson's equation by finite differences, J. Assoc. Comput. Mach. 12 (1965) 114-123. doi:10.1145/321250.321260 C414

[3] P.G. Ciarlet, Introduction to numerical linear algebra and optimisation, 1st English edition, Cambridge University Press, 1989. doi:10.2277/0521339847 C414

[4] L. Collatz, Bemerkungen zur fehlerabschätzung für das differnzenverfahren bei partiellen differentialgleichungen, Z. Angew. Math. Mech. 13 (1933) 56-57. doi:10.1002/zamm.19330130110 C415

[5] D. Greenspan, On the numerical solution of problems allowing mixed boundary conditions, J. Franklin Inst. 277 (1964) 11-30.

doi:10.1016/0016-0032(64)90035-3 C414, C419

[6] Z. Jomaa and C. Macaskill, The Shortley-Weller embedded finite-difference method for the 3D Poisson equation with mixed boundary conditions, submitted. C419

[7] Z. Jomaa and C. Macaskill, The embedded finite difference method for the Poisson equation in a domain with an irregular boundary and Dirichlet boundary conditions, J. Comp. Phys. 202 (2005) 488-506. doi:10.1016/j.jcp.2004.07.011 C419, C421 
[8] X. Liu, R. Fedkiw and M. Kang, A boundary condition capturing method for Poisson's equation on irregular domains, J. Comp. Phys. 160 (2000) 151-178. doi:10.1006/jcph.2000.6444 C414

[9] G. H. Shortley and R. Weller, The numerical solution of Laplace's equation, J. Appl. Phys. 9 (1938) 334-348. doi:10.1063/1.1710426 C415

\section{Author addresses}

1. Z. Jomaa, School of Maths \& Statistics, University of Sydney, Australia. mailto:ziadj@maths . usyd.edu . au

2. C. Macaskill, School of Maths \& Statistics, University of Sydney, Australia. 